\title{
Del offshore a la relocalización ventajas para el mejoramiento logístico en puerto y para las empresas logísticas
}

\section{From Offshore to relocation advantages for logistic improvement in port and for logistic companies}

Teófilo Omar Boyano-Fram

Universidad Libre - Colombia

ORCID iD: https://orcid.org/0000-0002-5373-4644

teofiloo.boyanof@unilibre.edu.co
Fecha de recepción: 03/05/2019

Fecha de evaluación: 17/07/2019

Fecha de aceptación: 05/02/2020

Cómo citar: Boyano-Fram, T. (2020). Del offshore a la relocalización ventajas para el mejoramiento logístico en puerto y para las empresas logísticas. Revista Cientifica Anfibios, 3(1), 60-67. https://doi.org/10.37979/afb.2020v3n1.63

\section{Resumen}

Con los grandes avances de la Gestión internacional para la movilidad del comercio exterior, no sólo los Puertos deben estar trabajando en la 4 y 5 Generación, también la manera de prestar el servicio es por eso que en este artículo se busca hacer una apuesta al offshore que facilita maniobras logísticas pero con la intencionalidad de mejorar los procesos de las naciones para la prestación y buen uso de sus empresas que puedan bajar costos, mejorar el servicio y simplificar aún más la movilidad, es decir perfeccionar su logística. En él se halló que la relocalización de las empresas se convierte en una posición logística competitiva y al utilizar plataformas offshore mejoran la movilidad en tiempo, aspecto muy importante para las empresas, los usuarios y los operadores o actores sociales de la maniobra logística.

Palabras claves:

Offshore; Ventajas; Mejoramiento logístico; Puertos; Empresas

\begin{abstract}
With the great advances of the international management for the mobility of the foreign trade, not only the Ports must be working in the 4 and 5 Generation, also the way to give the service is for that reason that in this article one looks for to make a bet to the offshore that facilitates logistic maneuvers but with the intention of improving the processes of the nations for the benefit and good use of its companies that can lower costs, improve the service and simplify still more the mobility, that is to say to perfect its logistic. It was found that the relocation of companies becomes a competitive logistic position and by using offshore platforms they improve mobility in time, a very important aspect for companies, users and operators or social actors of the logistic maneuver.
\end{abstract}

Keywords:

Offshore; Advantages; Logistics improvement; Ports; Businesses 


\section{Introducción}

El offshore es una construcción física que se hace en localidades que presentan morfologías naturales acuíferas, es decir, se hace en espacios arquitectónicos bien diseñados y construidos fuera de la plataforma continental, para facilitar las maniobras logísticas de explotación embarque y transporte de toda mercancía líquida en el caso de Colombia el petróleo y gaseosa como el gas, cualquiera sea su presentación, debe hacerse en un escenario propicio y óptimo para desarrollarlo.

Los países que lideran la exploración son Arabia Saudita, China y la India que se reportan hasta el momento alrededor de 530 plataformas activas en el mundo, correspondiendo a América

En Colombia se tiene conocimiento que, a finales del decenio de los años 70, más concretamente en el año de 1969 se dio inicio a la explotación en Manaure, Municipio al Norte de la Guajira con los campos denominados Chuchupa y el de Ballenas, este último uno de los más reconocidos y que hacen gran aporte a la producción de gas para el consumo en Colombia,

La relocalización o offshoring, ya es toda una apuesta de macro proyecto que permite a un Distrito, un Departamento o una Región a partir de una Política pública, favorecer la construcción de un polo de desarrollo de cualquier sector o subsector económico. Las empresas trasladan su filial o casa matriz a zonas que permitan la facilidad de la movilidad de sus mercancías y lo más cerca al Puerto.

La intención entonces es la de generar mayor valor competitivo a la organización exportando o importando productos que se puedan movilizar, en otras palabras, tengan posición logística que dé ventajas con respecto a sus competidores, para Colombia es una necesidad apremiante ya que es uno de los países que más peajes tiene en carreteras en cortas distancias. Por eso desde hace varios años se estudia la navegabilidad del Rio Magdalena, ya que en determinadas épocas del año es navegable en zonas de mayor profundidad, de allí la importancia, que el desarrollo logístico sea el más adecuado, que pueda generar el abastecimiento estratégico en Colombia. (Mendoza, 2016; Balza, 2019)

\section{Por qué el offshore es considerado una maniobra logística}

$\mathrm{Su}$ intencionalidad es facilitar la extracción o prestación de cualquier servicio, ya que le es más práctico y agiliza los procesos para la empresa, deja espacio para la movilidad en la plataforma continental o en tierra.

Respecto a ser un gran incentivo para la exoneración impuestos.

Aunque esto último que es la gestión de impuestos no debería ser la razón de ser la principal razón, algunas empresas deciden hacer uso para evitar otras maniobras logísticas que hacen incurrir en costos de operación. El cuidado de su gestión debe tenerse en cuenta; ya que un estudio que refleja la situación entre Nicaragua y Colombia en la disputa por el Archipiélago de San Andrés y específicamente sus aguas para la explotación, hecho por Gómez, G. P., \& Ribeiro, W. C. (2017) afirman que:

La búsqueda intensa de nuevos yacimientos o reservas se hace urgente para evitar mayores crisis del mercado. Sin embargo, con la llegada del pico las crisis comienzan a ser definitivas, esto impacta directamente precios y procesos de explotación, puesto que el afán por atenuar los problemas derivados de la escasez llevan a la implementación de procesos más agresivos con la naturaleza, de esta forma es que las explotaciones offshore de hidrocarburos se hacen más comunes, por tanto la búsqueda de ellos en el mar, y en muchos casos no se contempla el mar como una fuente de recursos alimenticios de poblaciones costeras pequeñas, ni los ecosistemas que deben ser preservados.

En ese caso es visto en primera instancia como un espacio, pero para la explotación de hidrocarburos, lo evidencia como una situación que debe alertar los posibles impactos ecológicos. Para la buena construcción se intentaría es tener el efecto contrario, es decir la explotación agua adentro con fines de mitigar consecuencias en la plataforma continental. Antes de entrar en funcionamiento la Plataforma debe cumplir con todas las Normas ISO o de Calidad, en especial las que verifican los procesos ambientales y el mapa de riesgos que señale como responder ante la comunidad de su entorno basado en una gestión socialmente responsable. (Navas \& González, 2016) 
Otro estudio relacionado con la exploración de gas por Fernández Tobar, Pablo (2012). Sostiene que:

El dimensionamiento del proceso de licuación de una planta offshore para la producción de gas natural licuado, usando únicamente $\mathrm{N} 2$ como refrigerante, evitando de este modo riesgos potenciales que podrían surgir con el uso de refrigerantes mixtos compuestos de hidrocarburos. El proceso ha sido diseñado para acomodar 35,23 $\mathrm{kg} / \mathrm{s}$ (aproximadamente un millón de toneladas por año) de gas natural seco, sin separación de gases licuados de petróleo (GLP) y ajustarlo dentro de los parámetros requeridos en las especificaciones del proceso. Para proceder al dimensionamiento del proceso de licuación de gas natural de la planta se ha empleado el programa Aspen Plus. Los sistemas floating production, storage and offloading para licuar el gas natural (LNG-FPSO), es una nueva unidad conceptual y un modo realista y efectivo para la explotación, recuperación, almacenamiento, transporte y agotamiento de los campos marginales de gas y las fuentes de gas asociadas offshore. En el proyecto se detalla el proceso, equipos necesarios y costes estimados, potencia aproximada requerida y un breve análisis económico.

Un estudio de caso que evidencia el accidente que se dio en el Golfo de México y cómo manejar los offshore adecuadamente, liderado por Pachón, C., Cristina, J., \& Martínez Vanegas, A. F. (2019) Aseguran que:

El accidente ocurrido en 2010, en la plataforma Deepwater Horizon con el pozo Macondo en el Golfo de México, brindando información para su contextualización tanto en su objetivo o target geológico como en su localización geográfica. Se realiza una compilación de información acerca del tipo de plataforma en la que se encontraba y se establecen los riesgos más representativos para una plataforma semisumergible; y finalmente se seleccionan y analizan mediante un análisis de causa raíz propuesto debido a que se realiza en diferentes esquemas inmersos del accidente del caso de estudio, como lo son la matriz RAM de evaluación de riesgos, un mapa de los hechos para especificar cada uno de los eventos ocurridos para llegar al accidente fatal, un diagrama de espina de pescado mostrando las causas y un diagrama de queso suizo para representar las barreras a las cuales se perdieron. Por último, debido a que Colombia está iniciando sus proyectos offshore, se realiza una recopilación de normativas para generar una serie de recomendaciones con base al análisis realizado para el caso Macondo.
Uno de los retos de seguir en la modalidad logística de Offshore en Colombia ha sido la baja de los precios de hidrocarburos en el mercado mundial, un estudio hecho por Castellanos, A., Lombana, J., \& Ortiz, M. (2017).

La industria de hidrocarburos en Colombia ha sido fuertemente afectada por la volatilidad internacional en los precios y la ausencia de nuevos campos de exploración/explotación. Ante la escasez del recurso y como fuente de ingresos para el territorio se ve como una alternativa la exploración/explotación aguas afuera (offshore). Para ello se deben dar transformaciones desde lo normativo hasta lo técnico, y hay ciudades como Barranquilla (Colombia) que ya están apostando por esa transformación local. El propósito del artículo es reflexionar, a partir del estado del arte de la experiencia offshore internacional, sobre brechas técnicas, normativas y académicas con Colombia, además plantear una estrategia logística que se derive en beneficios y en el reconocimiento de los aspectos críticos del tipo de explotación y de la transformación de la ciudad para acogerla. Se concluye que, a pesar de las brechas, las ciudades del Caribe, y en particular Barranquilla, están allanando el terreno para convertirse en una plataforma offshore de desarrollo logístico.

Sostiene que el momento apremia y que se hace necesario su modalidad para Colombia y la justifica ante la proyección de la exploración y es así como Visbal, J. P. B (2017)

En este trabajo se realiza un análisis de la situación actual y futura del desarrollo de la industria costa afuera (offshore) de hidrocarburos (petróleo y gas) en el Caribe Colombiano. Para el desarrollo de este artículo, se contó con información obtenida a través de la revisión de la literatura académica, científica y gubernamental contenida en diversas fuentes primarias y secundarias disponibles sobre el tema de estudio. Se parte de la base que con la cantidad de reservas probadas de petróleo y gas existentes en Colombia sería necesario buscar cómo suplir las necesidades para cubrir la demanda energética del país, debido a que las reservas actuales sólo dan suficiencia para un poco más de seis años para el petróleo y ocho años como máximo para el gas. Esto conlleva a buscar una opción diferente a la exploración y producción en tierra con el propósito de compensar el potencial desabastecimiento de hidrocarburos en el país para los próximos años; siendo esta opción el desarrollo de exploración y producción costa afuera en el Caribe Colombiano. 
En Europa un estudio relacionado con Offshore, pero para mejorar el aire ante tantos problemas ambientales en el mundo y para favorecer la energía eólica y la apuesta a un modelo específico, en España por De Prado González, I. (2018). sostiene que.

Considerando y realizando un análisis de los diferentes conceptos existentes acerca de plataformas flotantes para la industria offshore, debido a características como su alta estabilidad, relativamente sencilla construcción y las condiciones que simplifican en gran medida las tareas de instalación, operación y fondeo, se escoge el tipo Semi-sumergible o Semi-sub como la subestructura a emplear. Como modelo comercial de referencia, se toma la plataforma eólica flotante WindFloat, cuyos prototipos han sido probados obteniendo resultados satisfactorios en condiciones similares en la costa portuguesa. (pág. 159)

México tiene otro estudio relacionado con el problema de las Plataformas Offshore así que León-Escobedo, D. D. (2012).

Dadas las importantes consecuencias económicas de una falla de la plataforma petrolera, todos los aspectos de su comportamiento estructural y problemas de seguridad deben ser considerados cuidadosamente. En particular, los efectos de P-D en las patas de la cubierta de las plataformas marinas de la cubierta marina pueden ser relevantes cuando la altura de la plataforma y la carga vertical son significativas. En este documento, se examina y evalúa el impacto de la amplificación del momento, debido a la dureza de las patas de la plataforma, sobre la seguridad de la plataforma desde el punto de vista de la confiabilidad estructural. La formulación se aplica a una plataforma marina típica de cubierta alta bajo la carga ambiental en la Bahía de
Campeche, México, y su índice de confiabilidad se calcula con y sin el efecto P-D. Los resultados aquí presentados pueden ser mejorados para mejorar la práctica actual en el diseño y evaluación de plataformas marinas en alta mar en México y para actualizar la versión actual del código.

Las plataformas deben diseñarse de manera adecuada para soportar embates del Mar especialmente en la Zona del Golfo de México propensa a Huracanes de gran poder destructivo, mientras que otro estudio, en Santa Marta y en asocio con Petrobras el Puerto tiene una apuesta de Offshore para la explotación gasífera a partir del año 2017.

El terminal marítimo ha designado una zona dentro del Puerto, exactamente el muelle número 5, para uso exclusivo de Petrobras y su proyecto off shore, muelle donde la multinacional tendrá permanentemente lodo para perforación, silos para cemento, barita y bentonita, así como espacio para los equipos de exploración, los cuales serán cargados a los buques de suministro por medio de la grúa multipropósito Liebher que cuenta el Puerto. (SPR Santa Marta, 2017)

Con gran preocupación las voces de protestas aumentarán como se dio con la construcción de la Marina en el sector llamado La Playa en el Centro de la ciudad y que según sus moradores le quitó la vista paisajística a Punta Batín. No obstante, la generación de empleo y su vocación hacia el sector de servicios, el reto es interesante para que la ciudad siga creciendo. Barranquilla sigue también la apuesta en Plataformas Offshore específicamente en el Zona Franca de Palermo, con Repsol, Anadarko y Ecopetrol que son plataformas logísticas en Zona Franca y que darán empleo.

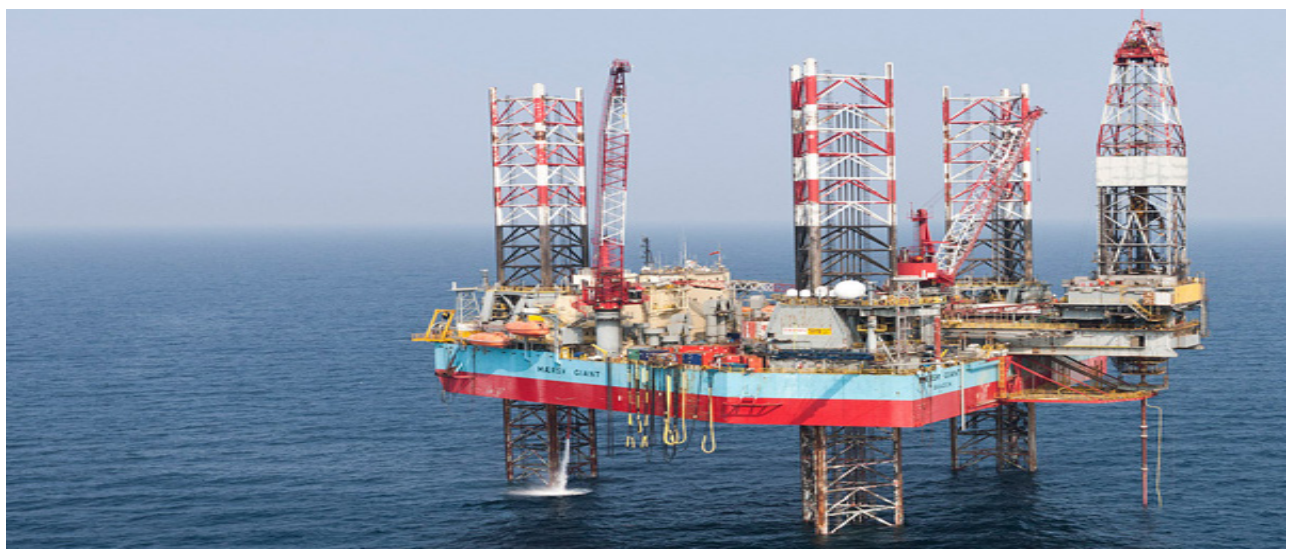

Imagen 1. Plataforma de perforación de Maersk.

Fuente: offshore-fleet.com (2017) 
Tabla 1. Las mejores plataformas Offshore en el mundo

\begin{tabular}{|c|c|c|c|}
\hline Nombre de la plataforma & Adquisiciones & Operación & Lugar de Servicios que ofrece \\
\hline $\begin{array}{l}1 \text { ENESCO (Arabia Sau- } \\
\text { dita) }\end{array}$ & $\begin{array}{l}\text { ARO drilling \& Sau- } \\
\text { di Aramco }\end{array}$ & $\begin{array}{l}82 \text { unidades: } \\
12 \text { plataformas semisumergibles. } \\
16 \text { buques de perforación. } \\
54 \text { jack ups } \\
\text { Opera en aguas profundas hasta } \\
1500 \text { metros bajo el nivel del mar }\end{array}$ & $\begin{array}{l}\text { Golfo de México Brasil } \\
\text { África Occidental } \\
\text { Mar del Norte } \\
\text { Mediterráneo } \\
\text { Oriente Medio } \\
\text { Sudeste asiático } \\
\text { Australia. }\end{array}$ \\
\hline 2.SEADRILL (Noruega) & $\begin{array}{l}\text { Odfjell, } \\
\text { Smedvig, } \\
\text { Eastern Drilling, } \\
\text { Scorpion Offshore, } \\
\text { North Atlantic Dri- } \\
\text { lling o Sevan Dri- } \\
\text { lling. }\end{array}$ & $\begin{array}{l}57 \text { unidades: } \\
29 \text { jack ups } \\
11 \text { buques de perforación } \\
17 \text { plataformas semisumergibles. } \\
\text { Con el Astillero de Cosco podrá } \\
\text { perforar hasta } 3.048 \text { metros de pro- } \\
\text { fundidad y perforar más de } 12.000 \\
\text { metros de profundidad }\end{array}$ & $\begin{array}{l}\text { Entre los sitios destacados está } \\
\text { Sur Corea y tiene presencia en } \\
\text { el } 605 \text { de plataformas en el } \\
\text { mundo }\end{array}$ \\
\hline $\begin{array}{l}\text { 3. Transocean- Ocean } \\
\text { Rig }\end{array}$ & $\begin{array}{l}\text { Southern Gar Com- } \\
\text { pany, } \\
\text { Sonat Offshore Dri- } \\
\text { lling } \\
\text { Transocean ASA, } \\
\text { Ocean Rig }\end{array}$ & $\begin{array}{l}57 \text { unidades } \\
31 \text { buques para aguas profundas } \\
\text { Líder en aguas profundas y turbu- } \\
\text { lentas }\end{array}$ & $\begin{array}{l}\text { En zonas turbulentas y profun- } \\
\text { das }\end{array}$ \\
\hline $\begin{array}{l}\text { 4. COSL } \\
\text { (China Oilfield Service } \\
\text { Limited) }\end{array}$ & Mantiene su nombre & $\begin{array}{l}32 \text { jack ups } \\
12 \text { plataformas semisumergibles }\end{array}$ & China \\
\hline $\begin{array}{l}\text { 5. Shelf drilling (Dubai- } \\
\text { Islas Caimán) }\end{array}$ & Mantiene su nombre & 38 jack ups (1) & $\begin{array}{l}\text { Asia } \\
\text { India } \\
\text { África Occidental } \\
\text { Medio Oriente } \\
\text { África septentrional } \\
\text { Mediterráneo }\end{array}$ \\
\hline 6. Borr Drilling & Mantiene su nombre & 36 jack-ups para el año 2020 & $\begin{array}{l}\text { Localizada en Islas Bermudas } \\
\text { y explora en aguas poco y me- } \\
\text { dianas profundas en gran parte } \\
\text { del mundo, tiene Astillero en } \\
\text { Singapur. Y Plataforma }\end{array}$ \\
\hline 7. Noble Corporation & Mantiene su nombre & $\begin{array}{l}28 \text { plataformas } \\
8 \text { buques de perforación } \\
6 \text { plataformas semi sumergibles y } 14 \\
\text { jack-ups }\end{array}$ & Londres y Texas \\
\hline 8. Maersk Drilling & $\begin{array}{l}\text { Filial de Ap Mollers } \\
\text { Maersk } \\
\text { Adquiere a Pacific } \\
\text { y Atlantic Marien } \\
\text { Corp. }\end{array}$ & $\begin{array}{l}15 \text { jack-ups } \\
4 \text { plataformas semi sumergibles } \\
4 \text { buques de perforación }\end{array}$ & $\begin{array}{l}\text { Copenhague y con más de } \\
4.000 \text { empleos }\end{array}$ \\
\hline 9. Adnoc Drilling & & 20 jack-ups & $\begin{array}{l}\text { Abu Dhabi National Oil Com- } \\
\text { pany }\end{array}$ \\
\hline 10. Abban Offshore & & $\begin{array}{l}15 \text { jack-ups } \\
2 \text { buques de perforación }\end{array}$ & $\begin{array}{l}\text { India, Malasia y Sudeste asiá- } \\
\text { tico. }\end{array}$ \\
\hline
\end{tabular}

Fuente: elaboración del propio autor 


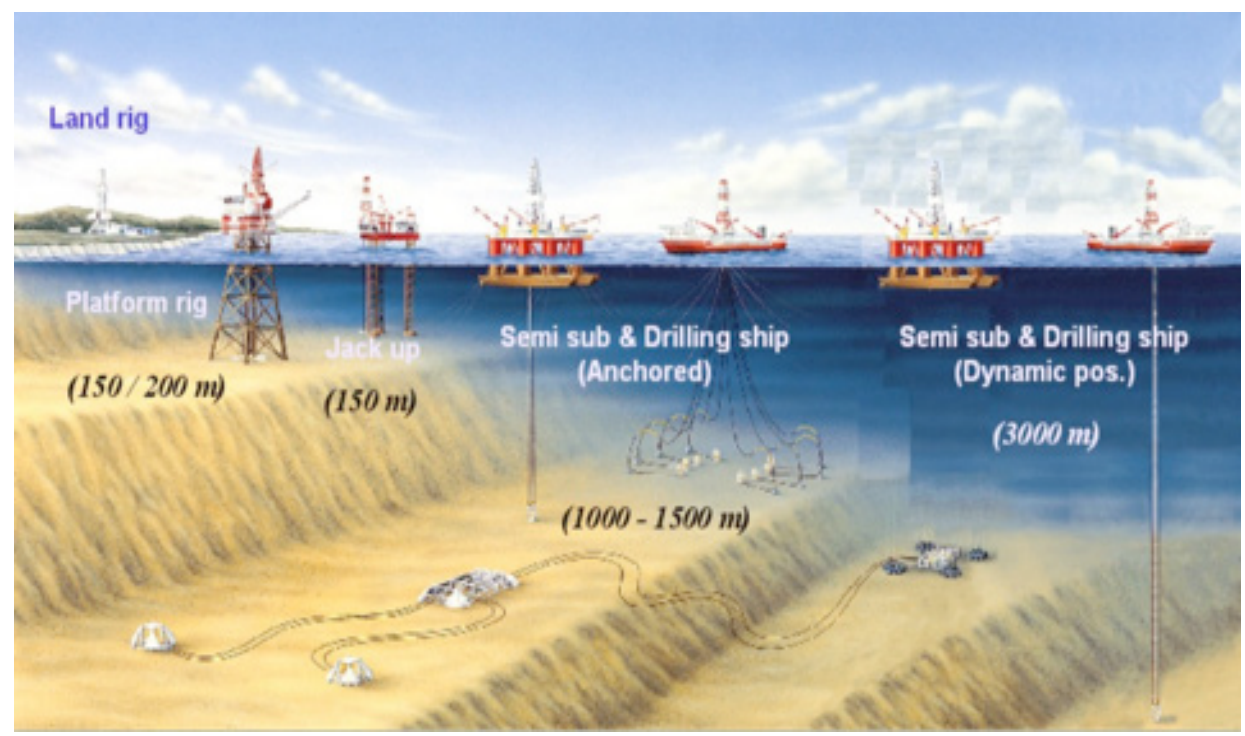

Imagen 2. Modalidades de offshore

Fuente: finanzasmania.com (2018)

Nótese que de acuerdo a la profundidad se construyen las plataformas offshore y de acuerdo a lo investigado cada empresa le apuesta a su modalidad, en todo caso se evidencia que se construyen hacia afuera o mar adentro.

A marzo de 2019 existen alrededor de 1039 plataformas, de las cuales la mayoría está en Oriente Medio por ser la zona de mayor producción de hidrocarburos, con 395 plataformas, luego viene Asia Pacífico con 234 y le sigue América Latina con 188 superando a Europa y África. Por países: China tiene 162, India 116 y Arabia Saudita 74; en América Latina México tiene 66, triplicando a Estados Unidos.

\section{Datos de Colombia en offshore}

En Colombia solamente se han perforado 9 pozos mar adentro desde el año 2010 a 2018, en parte desmotivado por la tendencia al a baja que tuvo el petróleo, según Mejía, M. I. (2016) en Colombia el aporte en divisas por parte del petróleo está en el 33\% del total de ingresos del país, y el 30\% de Inversión Extranjera Directa, y según el Departamento Nacional de Estadística. DANE representan el 5.5\% del Producto Interno Bruto. Existen 24 Contratos Offshore para El Caribe y 9 en estudio para una proyección de 33, y 12 para El Pacífico.

En síntesis, los retos del offshore conllevan a las naciones cambiar sus políticas públicas para la producción de bienes y servicios mar adentro, algunos se motivan por los atractivos fiscales, otros por la facilidad de operación cuando son expertos, ya que se recuerda que determinadas empresas invierten en zonas de gran turbulencia ya que los mares no tienen comportamientos iguales y en determinadas épocas del año las Estaciones y cambios climáticos impactan el sitio.

Llama la atención las nuevas modalidades de Offshore que se explora con la energía eólica en España, la tendencia de Colombia y América Latina en aprovechar más escenarios a pesar del grave accidente ocurrido en Golfo de México que trajo como consecuencia un impacto ambiental irreparable.

No descartarse otras plataformas con fines comerciales y nuevos productos, la apuesta estaría relacionada con la movilidad de los bienes, la facilidad de manipularlos y no deteriorar el entorno paisajístico y las comunidades.

\section{Conclusiones}

No puede negarse que para el caso de Colombia la Industria excepto en Cartagena de Indias específicamente la extractiva está localizada en zona Andina, generando sobrecostos en fletes y poca competitividad para el mercado externo, por lo que el Plan de Desarrollo de los próxi- 
mos Presidentes de Colombia deben procurar relocalizar empresas hacia el Caribe y hacia el Pacífico, el procesos debe ser bien planificado y que sea a largo plazo, ya que en 4 años de un gobierno sería poco lo que se avanzaría, pero en el largo plazo facilitaría la movilidad de la mercancía hacia afuera.

Empresas relocalizadas dan o generan mayor atractivo de inversión, generaría esa gestión más empleos y el país se vería con vocación más exportadora y la influencia en el Caribe actuaría como posición logística para los inversionistas y usuarios de esos bienes.

Se evitaría grandes inversiones de infraestructura vial, y ese costo de oportunidad se enfocaría a mejorar el Puerto para su competitividad, dada la gran seguridad de sus aguas y la Bahía que posee, unido a las nuevas Plataformas Offshore que se construirían.

\section{Referencias}

Balza-Franco, V., Paternina-Arboleda, C., \& Cardona-Arbeláez, D. (2019). Prácticas Colaborativas en la Cadena de Suministro: Una Revisión Conceptual. Saber, Ciencia y Libertad, 14(2), 77-101

Castellanos, A., Lombana, J., \& Ortiz, M. (2017). Exploración y explotación de hidrocarburos aguas afuera (offshore). Estrategia logística para Barranquilla, una ciudad en transformación. Equidad y Desarrollo, $1(28), 85-111$.

De Prado González, I. (2018). Estudio de implantación de un parque eólico offshore flotante en la costa de Cantabria (Doctoral dissertation, Universidad de Cantabria).

Fernández Tobar, Pablo (2012). Dimensionamiento del proceso de licuación de una planta de gas natural Offshore. Proyecto Fin de Carrera / Trabajo Fin de Grado, E.T.S.I. Minas (UPM)

Gómez, G. P., \& Ribeiro, W. C. (2017). Geopolítica y petróleo en el Mar Caribe: la tensión entre Colombia y Nicaragua Geopolitics of offshore oil exploration in the Caribbean Sea: border area between Colombia and Nicaragua. Revista Geográfica, (60).

León-Escobedo, D. D. (2012). P-D Effects on the Reliability of Oil Offshore Jacket Platforms in Mexico.

Mejía, M. I. (2016) Indicadores y Estrategias de Crecimiento del Sector de Hidrocarburos Colombiano. Agencia Nacional de Hidrocarburos. http://www.anh.gov.co/Sala-de-Prensa/Documents/ALAME\%20-\%20Colombia\%20Offshore.pdf

Mendoza Rivadeneira, M., \& Ceballos Polanco, N. (2016). El abastecimiento estratégico y su aplicación en las empresas. Saber, Ciencia Y Libertad, 11(1), 129-140. https://doi.org/10.18041/2382-3240/saber.2016v11n1.498

Offshore drilling. (2018). Introducción al análisis. http://www.finanzasmania.com/introduccion-al-analisis-offshore-drilling/

Offshore-fleet.com (2017). Jackup rig. Consultado 12 de enero de 2019. http://offshore-fleet.com/data/jackup-rig.htm

Navas Ríos, M., \& Romero-González, Z. (2016). Responsabilidad social universitaria. Saber, Ciencia Y Libertad, 11(1), 187-196. https://doi.org/10.18041/2382-3240/saber.2016v11n1.503

Pachón, C., Cristina, J., \& Martínez Vanegas, A. F. (2019). Análisis del influjo en el caso Macondo con el fin de generar recomendaciones para ser aplicadas en el caribe colombiano (Bachelor's thesis, Fundación Universidad de América). 
Sector Marítimo (2019) Documento recuperado en Revista del Sector Marítimo, Ingeniería Naval en https:// sectormaritimo.es/las-10-mayores-companias-de-perforacion-offshore-de-2018 España.

Visbal, J. P. B., Guillén, J. G. M., \& García, A. F. M. (2017) Perspectiva de la Industria Offshore de Hidrocarburos en el Caribe Colombiano. Visible In, 352. 